\title{
Combining Ability Analysis in Bt Cotton (Gossypium hirsutum L.) to Harness High Yield under Contrasting Planting Densities through Heterosis Breeding
}

\author{
Gowtham Prakash $^{1 *}$, S.L. Korekar ${ }^{2}$ and Sandeep Mankare ${ }^{2}$ \\ ${ }^{1}$ Department of Botany, Yeshwant Rao Chavan College, Tuljapur, Osmanabad, \\ Mahrashtra-413 601, India \\ ${ }^{2}$ Affiliated to, Dr. BAMU, Aurangabad, Maharashtra-431 004, India \\ *Corresponding author
}

\section{A B S T R A C T}

Biometric analysis was taken up to assess the combining ability of set of Bt cotton parents to harness high yield under contrasting planting densities through heterosis breeding. Twenty test crosses were generated out of Line (5) X Tester (4) mating design involving selected parents with diverse plant architecture. Field experimentation with randomized complete block design (RCBD) with three replicates was adopted to generate the quantitative data on maturity, plant architecture, yield components and key fibre quality

\section{Keywords}

Biometric, Line X Tester, Bt, Heterosis, Combining ability, Plant architecture

Article Info

Accepted:

17 January 2018 Available Online: 10 February 2018 traits under conventional density planting (CDP) and high density planting (HDP). The pooled analysis of variance for combining ability revealed significant line $\mathrm{x}$ tester interaction effect for all the traits under both planting densities whereas standalone line and tester effects displayed varying effects for array of traits. Proportion of $\mathrm{gca} / \mathrm{sca}$ variance found to be higher for traits such as days to $50 \%$ flowering, days to first boll opening, number of monopodial branches, ginning out turn, seed index, $2.5 \%$ span length, bundle strength and micronaire value indicating the preponderance of additive gene action. On contrary, for traits such as plant height, number of sympodial branches, boll weight, number of bolls per plant, seed cotton yield per plant recorded fractional values, indicating the prevalence of non-additive gene action. This phenomenon intern warrants for heterosis breeding as choice of method for genetic improvement of corresponding traits. Line SC1134 and tester SC1205 found to be good general combiner for the majority of the traits with broader utility, which could be explored as potential parents to breed for versatile hybrids for contrasting plant densities. And also identified, test cross combinations SC1104 X 1205 and SC1134 X SC1133 with high magnitude of sca effects under CDP and HDP respectively. These combinations can be considered as potential F1 hybrid crosses to achive high yield potential through heterosis breeding.

\section{Introduction}

Cotton (Gossypium hirsutum L.) is an important fibre crop and plays a vital role as a cash crop in Indian economy. Development of new variety with high yield and fibre quality is the primary objective of all cotton breeders. In breeding programs, analysis of general 
combining ability (GCA) and specific combining ability (SCA) are essential to identify best inbred lines for hybrid development and hybrid combinations with better specific combining ability. Vasal (1992) proposed the development of inbreds having high combining abilities has a fundamental role in the efficient exploitation of heterosis.

Combining ability is an effective tool which gives useful genetic information for the choice of parents in terms of their performance in series of crosses (Sprague and Tatum, 1942). If, GCA variances are found to be higher than SCA variance, then there is preponderance of additive gene action and in this case progeny selection will be effective for the genetic improvement of such traits, if SCA variances are found to be higher than GCA variances then there is preponderance of non-additive gene action and therefore heterosis breeding may be rewarding, but if both GCA and SCA variances are of equal magnitude it shows that both additive and non-additive gene action is equally important in the expression of characters. In such situations reciprocal recurrent selection may be resorted for population improvement.

India stood first with area 11.8 million hectors (33.23\% of world area), second in production and second in production with 34.6 million bales $(22.67 \%$ of world production as of $2015-$ 16. In terms of productivity levels, India has registered $495 \mathrm{~kg}$ lint/ha compared to average world average of $705 \mathrm{~kg}$ lint/ha (Anon., 2017) during the same time indicating wide gap and huge scope for genetic improvement and optimization of crop management practices. High density planting with compact determinate plant architecture is considered as one of the most important factors driving the high productivity levels in countries such as Brazil (1476 kg lint /ha), Turkey (1433 kg lint/ha) and China (1352 kg lint/ha). On contrary, majority of the hybrids commercialized so far in India are with indeterminate and semi determinate type readily adapting to relatively low plant densities. In order to facilitate the development of hybrids suitable for high density planting the present study has been taken up with to determine combining ability for plant architecture, yield, yield components and fibre quality traits under contrasting plant densities.

\section{Materials and Methods}

Nine parental lines with varying plant architecture (Table 1) were selected and sourced from M/S Sri Satya Agri Biotech Pvt. Ltd. Guntur germplasm. Twenty test hybrids were evolved in Line x Tester (Kempthrone, 1957) design by considering five $\mathrm{Bt}$ transgenic lines as females and non Bt lines as males through conventional hand emasculation and pollination.

Field experiment was conducted in the year2015-2016 at the research farm, Aurangabad with deep black cotton soil, situated at latitude $19^{\circ} 44^{\prime} \mathrm{N}$, longitude $73^{\circ} 59^{\prime}$ E. The study consisted of 29 entries such as 20 test hybrids and nine corresponding parental lines. Randomized block design involving three replicates was adopted to evaluate the test material, wherein each plot consisted of two rows of six meter length. To assess response of test hybrids with contrasting plant densities, paired trial was conducted with same design in higher density planting (HDP) and conventional density planting (CDP). Based on the previous studies, planting density of 37037 plants/ha @ 90X30 cm spacing with supplementary sprays of chloro mepiquat chloride (Lihocin ${ }^{\circledR} 0.2 \mathrm{ml} /$ litre of water)@60DAS + 90DAS and 18518 plants/ha @ 90x60cm spacing was considered as HDP and CDP respectively. The crop was raised under protected irrigation system with standard agronomic practices of the region. 
Data on key traits influencing maturity and plant architecture such as days to $50 \%$ flowering (DFF) and days to first boll opening (DFBO), plant height (PH), number of monopodial branches and number of sympodial branches. In order to assess the impact on yield traits such as number of bolls per plant (NBP), boll weight (BW), seed cotton yield per plant (SCYPP), ginning outrun (GOT) seed index (SI) was collected. Key fibre quality traits such as $2.5 \%$ Span length (SPL), bundle strength (BS) and Micronaire value (MC) were analyzed with HVI (High Volume Instrumentation) at central institute for research on cotton technology (CIRCOT), situated at Lam Farm, Guntur, Andhra Pradesh. Along with this seed cotton yield per hectare (SCYPH) which is considered as economic yield was worked out by extrapolating the gross plot yield $(\mathrm{kg})$. The data were analyzed for combining ability by following the method of Singh and Chaudhary (1979) by using the WINDOSTAT advanced biometric statistical package.

\section{Results and Discussion}

\section{Analysis of variance (ANOVA) for combining ability}

The pooled analysis of variance for combining ability (Table 2) revealed significant effects between the planting methods (PM). Hence separate analysis was performed and reported (Table 3, 4 and 5) for conventional density planting (CDP) and high density planting (HDP) methods similar to investigation of Gezahegn et al., (2010) in maize. The source of variance partitioned into components such as line (female) effect, tester (male) effect and line $\mathrm{x}$ tester effect to assess the impact. Significant line $x$ tester interaction effect was reported for all the traits under both planting densities whereas standalone line and tester displayed varying effects to array of traits in conjunction with planting densities. Testers (males) exerted consistent significant effects for traits such as days to $50 \%$ flowering, days to first boll opening, number of monopodial branches, boll weight, ginning out turn, bundle strength. Similarly lines (females) had significant impact on traits such as number of monopodial branches, boll weight, number of bolls per plant, ginning out turn, $2.5 \%$ span length and micronaire value. These findings are in agreement with Dhamayanthi et al., (2017) and Srinivas et al., (2014). The variance due to general combining ability (gca) and specific combining ability (sca) found to be significant for almost all the characters studied indicating both additive and non-additive gene action underlying the expression of traits. However the proportion $\mathrm{gca} / \mathrm{sca}$ variance found to be higher for traits such as days to $50 \%$ flowering, days to first boll opening, number of monopodial branches, ginning out turn, seed index, $2.5 \%$ span length, bundle strength and micronaire value indicating the preponderance of additive gene action. For these traits simple selection in early segregating generations could be considered in the breeding schemes.

On contrary, for traits such as plant height, number of sympodial branches, boll weight, number of bolls per plant, seed cotton yield per plant recorded lower proportion indicting the prevalence of non-additive gene action. This phenomenon warrants for the heterosis breeding in order to exploit these traits. In addition to mean sum of squares effects and combining ability variance, percent contribution of lines (females), testers (males) and line $\mathrm{x}$ testers towards variance was enumerated to access the relative impact on the manifestation of traits under the study. Wide variation was observed in terms of contribution by lines, testers and line $\mathrm{x}$ testers across for the array of traits under contrasting planting densities. This necessitates the specific attention to the targeted traits for the genetic improvement. 
Table.1 Salient features of parental lines used for the development of test crosses

\begin{tabular}{|c|c|c|c|c|l|c|}
\hline SI. No. & Line/Tester & Name & Bt/Non Bt & Plant type & \multicolumn{1}{|c|}{ Growth pattern } & Boll size \\
\hline $\mathbf{1}$ & Line & SC1104 & Bt & Bushy & Indeterminate (ID) & Big \\
\hline $\mathbf{2}$ & Line & SC1112 & $\mathrm{Bt}$ & Open & Semi determinate (SD) & Medium \\
\hline $\mathbf{3}$ & Line & SC1117 & $\mathrm{Bt}$ & Semi open & Semi determinate (SD) & Medium \\
\hline $\mathbf{4}$ & Line & SC1132 & $\mathrm{Bt}$ & Semi open & Semi determinate (SD) & Big \\
\hline $\mathbf{5}$ & Line & SC1134 & $\mathrm{Bt}$ & Open & Determinate (D) & Small \\
\hline $\mathbf{6}$ & Tester & SC1115 & Non Bt & Bushy & Indeterminate (ID) & Small \\
\hline $\mathbf{7}$ & Tester & SC1133 & Non Bt & Semi open & Semi determinate (SD) & Big \\
\hline $\mathbf{8}$ & Tester & SC1205 & Non Bt & Open & Determinate (D) & Medium \\
\hline $\mathbf{9}$ & Tester & SC1206 & Non Bt & Open & Extremely Determinate (ED) & Small \\
\hline
\end{tabular}

Table. 2 Pooled analysis of variance involving conventional density planting (CDP) and high density plating (HDP) methods for all the traits

\begin{tabular}{|c|c|c|c|c|c|c|c|c|c|c|}
\hline \multicolumn{11}{|c|}{ Source of variation } \\
\hline Traits & Replication & $\begin{array}{l}\text { Planting methods } \\
\text { (PM) }\end{array}$ & $\begin{array}{l}\text { Replication } \mathrm{x} \\
\text { PM }\end{array}$ & $\begin{array}{l}\text { Line (female) } \\
\text { effect }\end{array}$ & $\begin{array}{l}\text { Tester (male) } \\
\text { effect }\end{array}$ & $\begin{array}{l}\text { Line } x \text { Tester } \\
\text { effect }\end{array}$ & $\begin{array}{l}\text { PM x Line } \\
\text { effect }\end{array}$ & $\begin{array}{l}\text { PM x Tester } \\
\text { effect }\end{array}$ & $\begin{array}{l}\text { PM x Line x Tester } \\
\text { effect }\end{array}$ & $\begin{array}{l}\text { Pooled } \\
\text { error }\end{array}$ \\
\hline df & 2 & 1 & 2 & 4 & 3 & 12 & 4 & 3 & 12 & 112 \\
\hline DFF & 1.3 & $18.7 * *$ & 0.5 & $17.2 *$ & $189.4 * *$ & $4.9^{* *}$ & 2.1 & 2.9 & $2.6^{* *}$ & 0.9 \\
\hline DFBO & 0.9 & $2.1 *$ & 0.09 & $137.4^{*}$ & $518.4 * *$ & $41.4^{* *}$ & 1.8 & $3.4^{*}$ & $0.7 *$ & 0.4 \\
\hline PH & 11.0 & $62890.0 * *$ & 2.7 & 121.3 & $485.0^{*}$ & $123.0^{* * *}$ & 146.9 & 147.2 & $66.2^{* *}$ & 1.0 \\
\hline NSB & 0.20 & $228.51 * *$ & 0.00 & 4.51 & 3.95 & $2.84 * *$ & 2.07 & 3.63 & $1.22 * *$ & 0.03 \\
\hline NMB & 0.01 & $26.26^{* *}$ & 0.00 & $3.06 * *$ & $16.83 * *$ & $0.12 * *$ & 0.06 & 0.21 & $0.13^{* *}$ & 0.02 \\
\hline NBP & 5.0 & $4035.9 * *$ & 0.11 & $411.2 *$ & 332.1 & $120.0^{* *}$ & $54.6 * *$ & $162.9 * *$ & $3.3 * *$ & 1.3 \\
\hline BW & 0.02 & $8.36^{* *}$ & 0.01 & $2.86^{* *}$ & $2.92 * *$ & $0.25^{* *}$ & 0.21 & 0.10 & $0.06^{* *}$ & 0.01 \\
\hline SCYPP & 15.9 & $34977.5^{* *}$ & 8.7 & 614.0 & 737.7 & $388.5^{* *}$ & $843.4^{*}$ & 1007.0* & $218.9 * *$ & 9.2 \\
\hline SCYPH & 6035.0 & $88913114.5^{* *}$ & 2131.2 & 361694.4 & $1087801.0^{*}$ & $249295.9 * *$ & $512356.5^{*}$ & $1255213.7 * *$ & $133740.5^{* * *}$ & 6742.9 \\
\hline GOT & 0.10 & $64.09 * *$ & 0.24 & $25.62 * *$ & $101.47 * *$ & $3.34 * *$ & $2.30 *$ & 0.42 & $0.62 * *$ & 0.10 \\
\hline SI & 0.35 & $56.33^{* *} *$ & 0.12 & $18.97 * *$ & $13.97^{*}$ & $3.08 * *$ & 2.34 & 6.06 & $1.99 * *$ & 0.07 \\
\hline SPL & 0.33 & $9.10^{* *}$ & 0.00 & $13.71 * *$ & $34.39 * *$ & $0.98^{* *}$ & 0.37 & 2.64 & $1.44 * *$ & 0.04 \\
\hline BS & 0.02 & $19.47 * *$ & 0.21 & $5.92 * *$ & $23.43 * *$ & $0.70^{* * *}$ & 1.66 & 1.60 & $1.20 * *$ & 0.14 \\
\hline MV & 0.13 & $3.65^{* *}$ & 0.00 & $1.55^{* * *}$ & $0.76^{* *}$ & $0.12 * *$ & 0.28 & 0.29 & $0.13^{* *}$ & 0.01 \\
\hline
\end{tabular}

Table.3 Combining ability ANOVA for maturity and plant architecture traits under contrasting plant densities

\begin{tabular}{|c|c|c|c|c|c|c|c|c|c|c|c|}
\hline \multirow[t]{2}{*}{ Source of variation } & \multirow[t]{2}{*}{ df } & \multicolumn{2}{|c|}{ DFF } & \multicolumn{2}{|c|}{ DFBO } & \multicolumn{2}{|c|}{ PH } & \multicolumn{2}{|c|}{ NSB } & \multicolumn{2}{|c|}{ NMB } \\
\hline & & CDP & HDP & CDP & HDP & CDP & HDP & CDP & HDP & CDP & HDP \\
\hline Replication & 2 & 0.9 & 1.1 & 0.4 & 0.8 & 6.0 & 17.0 & 0.21 & 0.06 & 0.02 & 0.00 \\
\hline Line effect (female) & 4 & 8.7 & $10.6 * *$ & $81.6^{*}$ & 57.6 & 31.9 & 236.4 & $5.33 * *$ & 1.24 & $1.68 * *$ & $1.43^{* *}$ \\
\hline Tester effect (male) & 3 & $104.1^{* *}$ & $88.1 * *$ & $226.1 * *$ & $295.7 * *$ & 235.1 & $397.1^{*}$ & $5.38 * *$ & 2.20 & $10.35^{* *}$ & $6.68^{* *}$ \\
\hline Line $x$ Tester effect & 12 & $5.9^{* *}$ & $1.6^{*}$ & $21.8^{* *}$ & $20.3^{* *}$ & $98.5 * *$ & $90.8^{* * *}$ & $0.47 * *$ & $3.58 * *$ & $0.14 * *$ & $0.10^{* *}$ \\
\hline Error & 38 & 2.1 & 0.2 & 0.7 & 0.2 & 0.2 & 1.1 & 0.03 & 0.04 & 0.02 & 0.01 \\
\hline \multicolumn{12}{|l|}{ Variance } \\
\hline$\sigma^{2}$ gca & & 4.07 & 3.64 & 11.35 & 13.07 & 9.88 & 23.33 & 0.15 & 0.13 & 0.44 & 0.30 \\
\hline$\sigma^{2}$ sea & & 1.46 & 1.45 & 7.06 & 6.72 & 32.79 & 29.65 & 0.39 & 1.19 & 0.04 & 0.03 \\
\hline$\sigma^{2} \mathrm{gca} / \sigma^{2} \mathrm{sca}$ & & 2.79 & 2.51 & 1.61 & 1.95 & 0.30 & 0.79 & 0.39 & 0.11 & 10.83 & 9.09 \\
\hline \multicolumn{12}{|l|}{$\%$ Contribution } \\
\hline Lines (females) & & 8.3 & 13.0 & 25.8 & 16.9 & 6.3 & 29.3 & 49.5 & 9.1 & 17.0 & 21.1 \\
\hline Testers (males) & & 74.7 & 81.3 & 53.6 & 65.2 & 35.0 & 36.9 & 37.4 & 12.1 & 78.6 & 74.0 \\
\hline Lines X Testers & & 17.0 & 5.7 & 20.6 & 17.9 & 58.7 & 33.8 & 13.1 & 78.8 & 4.4 & 4.9 \\
\hline
\end{tabular}


Table.4 Combining ability ANOVA for yield and yield component traits under contrasting plant densities

\begin{tabular}{|c|c|c|c|c|c|c|c|c|c|}
\hline \multirow[t]{2}{*}{ Source of variation } & \multirow[t]{2}{*}{ df } & \multicolumn{2}{|c|}{ BW } & \multicolumn{2}{|c|}{ NBP } & \multicolumn{2}{|c|}{ SCYPP } & \multicolumn{2}{|c|}{ SCYPH } \\
\hline & & CDP & HDP & CDP & HDP & CDP & HDP & CDP & HDP \\
\hline Replication & 2 & 0.01 & 0.02 & 1.0 & 0.32 & 65.6 & 66.6 & 22268.1 & 7860.7 \\
\hline Line (female) effect & 4 & $0.93 * *$ & $2.14 * *$ & $177.0^{*}$ & $288.9^{*}$ & 1084.2 & 373.3 & 371081.2 & 502970.1 \\
\hline Tester (male) effect & 3 & $1.10 * *$ & $1.91 * *$ & 61.1 & $433.9 * *$ & 27.8 & $1716.9 * *$ & 8325.6 & $2334689.0 * *$ \\
\hline Line $x$ Tester effect & 12 & $0.05 * *$ & $0.27 * *$ & $53.7 * *$ & $69.6^{* *}$ & $436.6 * *$ & $170.7 * *$ & $150012.1 * *$ & $233024.3 * *$ \\
\hline Error & 38 & 0.001 & 0.02 & 0.6 & 1.9 & 7.2 & 9.4 & 2390.4 & 12460.1 \\
\hline \multicolumn{10}{|l|}{ Variance } \\
\hline$\sigma^{2}$ gca & & 0.02 & 0.08 & 8.78 & 22.46 & 40.39 & 54.39 & 13781 & 74400 \\
\hline$\sigma^{2} \operatorname{sca}$ & & 0.08 & 0.15 & 17.75 & 26.61 & 141.94 & 76.85 & 48784 & 104371 \\
\hline$\sigma^{2} \mathrm{gca} / \sigma^{2}$ sca & & 0.25 & 0.53 & 0.50 & 0.85 & 0.28 & 0.71 & 0.28 & 0.71 \\
\hline \multicolumn{10}{|l|}{$\%$ Contribution } \\
\hline Lines (females) & & 48.5 & 48.9 & 46.1 & 35.1 & 44.9 & 17.1 & 44.9 & 17.0 \\
\hline Testers (males) & & 43.3 & 32.9 & 11.9 & 39.5 & 0.9 & 59.3 & 0.8 & 59.3 \\
\hline Lines X Testers & & 8.2 & 18.2 & 42.0 & 25.4 & 54.2 & 23.6 & 54.3 & 23.7 \\
\hline
\end{tabular}

Table.5 Combining ability ANOVA for yield and fibre quality traits under contrasting plant densities

\begin{tabular}{|c|c|c|c|c|c|c|c|c|c|c|c|}
\hline \multirow[t]{2}{*}{ Source of variation } & \multirow[t]{2}{*}{ df } & \multicolumn{2}{|c|}{ GOT } & \multicolumn{2}{|c|}{ SI } & \multicolumn{2}{|c|}{ SPL } & \multicolumn{2}{|c|}{ BS } & \multicolumn{2}{|c|}{ MV } \\
\hline & & CDP & HDP & CDP & HDP & CDP & HDP & CDP & HDP & CDP & HDP \\
\hline Replication & 2 & 0.11 & 0.17 & 0.26 & 0.12 & 0.09 & 0.15 & 0.06 & 0.16 & 0.03 & 0.02 \\
\hline Line effect (female) & 4 & $9.25 * *$ & $18.67 * *$ & $10.51 * *$ & 10.79 & $8.44 * *$ & $5.64 * *$ & $6.00 * *$ & 1.58 & $1.12 * *$ & $0.70 * *$ \\
\hline Tester effect (male) & 3 & $52.51 * *$ & $49.37 * *$ & $16.93 * *$ & 3.09 & $21.50 * *$ & $15.52 * *$ & $9.67 * *$ & $15.34 * *$ & 0.54 & $0.51 * *$ \\
\hline Line $x$ Tester effect & 12 & $1.17 * *$ & $2.77 * *$ & $1.51 * *$ & $3.54 * *$ & $1.42 * *$ & $0.99 * *$ & $0.47 * *$ & $1.42 * *$ & $0.17 *$ & $0.07 *$ \\
\hline Error & 38 & 0.13 & 0.03 & 0.06 & 0.08 & 0.01 & 0.07 & 0.17 & 0.17 & 0.02 & 0.01 \\
\hline \multicolumn{12}{|l|}{ Variance } \\
\hline$\sigma^{2}$ gca & & 2.28 & 2.52 & 1.01 & 1.15 & 1.11 & 0.78 & 0.57 & 0.62 & 0.06 & 0.05 \\
\hline$\sigma^{2}$ sca & & 0.34 & 0.92 & 0.49 & 0.51 & 0.47 & 0.31 & 0.11 & 0.43 & 0.05 & 0.02 \\
\hline$\sigma^{2} \mathrm{gca} / \sigma^{2} \mathrm{sca}$ & & 6.75 & 2.75 & 2.07 & 2.26 & 2.35 & 2.51 & 1.18 & 1.96 & 1.18 & 1.96 \\
\hline \multicolumn{12}{|l|}{$\%$ Contribution } \\
\hline Lines (females) & & 17.7 & 29.2 & 37.9 & 45.4 & 29.3 & 27.9 & 40.8 & 9.1 & 54.5 & 53.6 \\
\hline Lines X Testers & & 6.8 & 13.0 & 16.4 & 45.0 & 14.8 & 14.7 & 9.8 & 24.7 & 25.8 & 17.0 \\
\hline
\end{tabular}


Table.6 Top female and male parents with high general combining ability (GCA) effects for all the traits under contrasting plant densities

\begin{tabular}{|c|c|c|c|c|c|c|c|c|}
\hline \multirow{3}{*}{ Traits } & \multicolumn{4}{|c|}{ Conventional density planting (CDP) } & \multicolumn{4}{|c|}{ High density planting (HDP) } \\
\hline & \multicolumn{2}{|c|}{ Females (lines) } & \multicolumn{2}{|c|}{ Male (Tester) } & \multicolumn{2}{|c|}{ Females (lines) } & \multicolumn{2}{|c|}{ Male (Tester) } \\
\hline & Code & GCA effect & Code & GCA effect & Code & GCA effect & Code & GCA effect \\
\hline DFF & SC1104 & -1.00 & SC1206 & -3.32 & SC1134 & -1.37 & SC1206 & -3.10 \\
\hline DFBO & SC1134 & -1.37 & SC1206 & -4.88 & SC1134 & -2.82 & SC1206 & -5.63 \\
\hline PH & SC1117 & 0.97 & SC1205 & 3.33 & SC1132 & -7.32 & SC1133 & -6.55 \\
\hline NSB & SC1134 & 1.14 & SC1206 & 0.50 & SC1112 & 0.38 & SC1133 & 0.53 \\
\hline NMB & SC1134 & -0.50 & SC1206 & -0.90 & SC1134 & -0.51 & SC1206 & -0.73 \\
\hline NBP & SC1134 & 7.50 & SC1205 & 4.95 & SC1134 & 6.26 & SC1205 & 3.90 \\
\hline BW & SC1132 & 0.44 & SC1115 & 0.29 & SC1132 & 0.55 & SC1115 & 0.46 \\
\hline SCYPP & SC1104 & 11.58 & SC1115 & 1.15 & SC1134 & 5.85 & SC1205 & 10.80 \\
\hline SCYPH & SC1104 & 212.9 & SC1115 & 21.43 & SC1134 & 212.7 & SC1205 & 396.3 \\
\hline GOT & SC1117 & 1.11 & SC1205 & 1.69 & SC1134 & 0.97 & SC1205 & 1.69 \\
\hline SI & SC1134 & -1.02 & SC1205 & -1.33 & SC1112 & -1.00 & SC1205 & -0.43 \\
\hline SPL & SC1112 & 0.90 & SC1115 & 1.45 & SC1112 & 0.70 & SC1115 & 0.97 \\
\hline BS & SC1104 & 0.70 & SC1115 & 1.08 & SC1112 & 0.37 & SC1115 & 0.99 \\
\hline $\mathbf{M V}$ & SC1134 & -0.54 & SC1205 & -0.23 & SC1134 & -0.28 & SC1115 & -0.15 \\
\hline
\end{tabular}

Table.7 Relative GCA (General combining ability) scores of female and male parents in significantly favorable and non-favorable direction for all the traits under contrasting plant densities

\begin{tabular}{|c|c|c|c|c|c|c|}
\hline \multirow{2}{*}{ Parents } & \multicolumn{3}{|c|}{ Conventional density planting (CDP) } & \multicolumn{3}{|c|}{ High density planting (HDP) } \\
\hline & $\begin{array}{l}\text { Significantly } \\
\text { favorable } \\
\text { direction }\end{array}$ & $\begin{array}{l}\text { Significantly non } \\
\text { favorable direction }\end{array}$ & $\begin{array}{l}\text { Non- } \\
\text { significant }\end{array}$ & $\begin{array}{l}\text { Significantly } \\
\text { favorable } \\
\text { direction }\end{array}$ & $\begin{array}{c}\text { Significantly non } \\
\text { favorable } \\
\text { direction }\end{array}$ & $\begin{array}{l}\text { Non- } \\
\text { significant }\end{array}$ \\
\hline \multicolumn{7}{|c|}{ Lines (Females) } \\
\hline SC1104 & 7 & 6 & 1 & 5 & 6 & 3 \\
\hline SC1112 & 7 & 4 & 3 & 8 & 4 & 2 \\
\hline SC1117 & 5 & 7 & 2 & 2 & 9 & 3 \\
\hline SC1132 & 3 & 7 & 4 & 5 & 7 & 2 \\
\hline SC1134 & 8 & 2 & 4 & 9 & 3 & 2 \\
\hline \multicolumn{7}{|c|}{ Testers (Males) } \\
\hline SC1115 & 4 & 8 & 2 & 4 & 9 & 1 \\
\hline SC1133 & 2 & 9 & 3 & 4 & 9 & 1 \\
\hline SC1205 & 10 & 2 & 2 & 9 & 4 & 1 \\
\hline SC1206 & 7 & 3 & 4 & 9 & 3 & 2 \\
\hline
\end{tabular}


Table.8 Specific combining ability (SCA) effects in desirable direction for top three crosses along with general combining ability (GCA) status of parents for maturity, phenological and yield component traits

\begin{tabular}{|c|c|c|c|c|c|c|c|c|}
\hline \multirow[t]{2}{*}{ Traits } & \multicolumn{4}{|c|}{ Conventional density planting (CDP) } & \multicolumn{4}{|c|}{ High density planting (HDP) } \\
\hline & Crosses & Mean & SCA effect & $\begin{array}{c}\text { Parental } \\
\text { GCA status* }\end{array}$ & Crosses & Mean & SCA effect & $\begin{array}{c}\text { Parental } \\
\text { GCA status* }\end{array}$ \\
\hline \multirow[t]{3}{*}{ DFF } & SC1104 X 1206 & 54.67 & -2.60 & (-)High X (-)High & SC1117 X 1133 & 64.67 & -0.77 & $(+)$ High X (+)High \\
\hline & SC1132 X 1133 & 61.67 & -1.77 & (-)Low X (+)High & SC1104 X 1206 & 57.67 & -0.73 & (-)High X (-)High \\
\hline & SC1134 X 1133 & 62.00 & -1.02 & $(-)$ Low X (+)High & SC1132 X 1206 & 58.67 & -0.73 & (+)High X(-)High \\
\hline \multirow[t]{3}{*}{ DFBO } & SC1134 X 1206 & 107.00 & -5.53 & (-)High X (-)High & SC1134X 1206 & 107.67 & -4.78 & (-)High X (-)High \\
\hline & SC1112 X 1133 & 124.67 & -2.02 & (+)High X (+)High & SC1117 X 1133 & 123.67 & -2.88 & (+)High X (+)High \\
\hline & SC1104 X 1206 & 112.67 & -1.70 & (-)High X (-)High & SC1112 X 1133 & 124.67 & -2.05 & (+)High X (+)High \\
\hline \multirow[t]{3}{*}{$\mathbf{P H}$} & SC1104 X 1206 & 166.00 & 8.04 & (-)High X (+)High & SC1132 X 1206 & 100.50 & -7.06 & $(-)$ High X (+)Low \\
\hline & SC1104 X 1133 & 156.33 & 7.52 & (-)High X (-)High & SC1132 X 1205 & 106.70 & -6.54 & $(-)$ High $\mathrm{X}(+)$ High \\
\hline & SC1132 X 1205 & 162.90 & 5.60 & (+)High X (+)High & SC1117 X 1205 & 118.30 & -4.98 & (+)High X (+)High \\
\hline \multirow[t]{3}{*}{ NSB } & SC1134 X 1206 & 19.33 & 0.54 & (+)High X (+)High & SC1132 X 1206 & 16.17 & 1.76 & $(-)$ Low X (+)Low \\
\hline & SC1132 X 1206 & 18.10 & 0.53 & (-)Low X (+)High & SC1104 X 1133 & 15.77 & 1.27 & (-)High X (+)High \\
\hline & SC1104 X 1133 & 16.77 & 0.40 & (-)High X (-)High & SC1134 X 1206 & 15.50 & 0.86 & (+)High X (+)Low \\
\hline \multirow[t]{3}{*}{ NMB } & SC1112 X 1206 & 0.70 & -0.34 & (-)High X (-)High & SC1112 X 1133 & 2.00 & -0.23 & (-)Low X (+)High \\
\hline & SC1132 X 1206 & 1.00 & -0.29 & (+)Low X (-)High & SC1132 X 1206 & 0.50 & -0.19 & (+)Low X (-)High \\
\hline & SC1134 X 1115 & 1.70 & -0.17 & (-)High X (+)High & SC1134 X 1206 & 0.00 & -0.17 & (-)High X (-)High \\
\hline \multirow[t]{3}{*}{ NBP } & SC1117 X 1115 & 54.00 & 7.38 & (-)High X (-)High & SC1117 X 1115 & 43.67 & 7.95 & (-)High X (-)High \\
\hline & SC1112 X 1133 & 52.00 & 5.03 & (+)High X (-)High & SC1112 X 1133 & 41.00 & 7.70 & (-)Low X (-)High \\
\hline & SC1132 X 1206 & 47.00 & 3.93 & (-)High X (+)High & SC1104 X 1205 & 46.00 & 4.80 & (-)Low X (+)High \\
\hline \multirow[t]{3}{*}{$\mathbf{B W}$} & SC1134 X 1133 & 4.73 & 0.18 & (-)Low X (-)High & SC1112 X 1115 & 6.22 & 0.40 & (-)High X (+)High \\
\hline & SC1132 X 1133 & 5.44 & 0.15 & (+)High X (-)High & SC1134 X 1133 & 5.17 & 0.40 & (-)High X (-)High \\
\hline & SC1112 X 1115 & 5.49 & 0.14 & (-)Low X (+)High & SC1117 X 1205 & 5.91 & 0.27 & (-)Low X (+)High \\
\hline \multirow[t]{3}{*}{ SCYPP } & SC1117 X 1133 & 165.00 & 15.75 & (+)High X (+)Low & SC1134 X 1133 & 117.33 & 10.28 & (+)High X (-)High \\
\hline & SC1104 X 1206 & 167.00 & 14.75 & (+)High X (-)Low & SC1132 X 1206 & 121.67 & 9.37 & (-)High X (+)High \\
\hline & SC1104 X 1205 & 168.00 & 13.82 & (+)High X (+)Low & SC1112 X 1133 & 112.67 & 7.78 & (+)High X (-)High \\
\hline $\begin{array}{l}\text { *Female } \\
\text { DFF: Day } \\
\text { Plant heig }\end{array}$ & Inle dirention ( , & & & & & & & \\
\hline
\end{tabular}


Table.9 Specific combining ability (SCA) effects in desirable direction for top three crosses along with general combining ability (GCA) status of parents for yield and fibre quality traits

\begin{tabular}{|c|c|c|c|c|c|c|c|c|}
\hline \multirow[t]{2}{*}{ Traits } & \multicolumn{4}{|c|}{ Conventional density planting (CDP) } & \multicolumn{4}{|c|}{ High density planting (HDP) } \\
\hline & Crosses & Mean & SCA effect & $\begin{array}{c}\text { Parental } \\
\text { GCA status* }\end{array}$ & Crosses & Mean & SCA effect & $\begin{array}{c}\text { Parental } \\
\text { GCA status* }\end{array}$ \\
\hline \multirow[t]{3}{*}{ SCYPH } & SC1117 X 1133 & 3056.0 & 294.4 & $(+)$ High X (+)Low & SC1134 X 1133 & 4343.3 & 382.2 & (+)High X (-)High \\
\hline & SC1104 X 1206 & 3095.0 & 275.1 & (+)High X (-)Low & SC1132 X 1206 & 4509.0 & 345.6 & (-)High X (+)High \\
\hline & SC1104 X 1205 & 3108.0 & 255.2 & $(+)$ High X (+)Low & SC1112 X 1133 & 4168.3 & 282.0 & (+)High X (-)High \\
\hline \multirow[t]{3}{*}{ GOT } & SC1117 X 1205 & 40.40 & 0.77 & (+)High X (+)High & SC1134 X 1115 & 35.67 & 1.19 & (+)High X (-)High \\
\hline & SC1132 X 1133 & 35.67 & 0.74 & (-)High X (-)High & SC1104 X 1133 & 35.90 & 1.15 & (-)High X (-)High \\
\hline & SC1134 X 1115 & 35.40 & 0.60 & (+)Low X (-)High & SC1132 X 1133 & 34.10 & 1.06 & (-)High X (-)High \\
\hline \multirow[t]{3}{*}{ SI } & SC1104 X 1206 & 9.67 & -1.32 & (+)High X (+)High & SC1112 X 1115 & 8.00 & -1.80 & (-)High X (+)High \\
\hline & SC1112 X 1115 & 8.00 & -0.85 & (-)High X (+)High & SC1104 X 1133 & 9.00 & -1.63 & (+)High X (-)Low \\
\hline & SC1134 X 1133 & 7.00 & -0.58 & (-)High X (-)High & SC1132 X 1205 & 10.00 & -1.07 & (+)High X (-)High \\
\hline \multirow[t]{3}{*}{ SPL } & SC1104 X 1205 & 32.50 & 1.10 & (+)High X (-)High & SC1117 X 1133 & 33.70 & 1.13 & (-)High X (+)High \\
\hline & SC1134 X 1133 & 33.67 & 0.74 & (+)High X (+)High & SC1134 X 1206 & 32.30 & 0.59 & (-)Low X (-)High \\
\hline & SC1117 X 1115 & 33.20 & 0.71 & (-)High X (+)High & SC1104 X 1206 & 32.57 & 0.51 & (+)High X (-)High \\
\hline \multirow[t]{3}{*}{ BS } & SC1104 X 1133 & 25.50 & 0.56 & (+)High X (+)Low & SC1134 X 1206 & 26.70 & 1.02 & (-)High X (-)High \\
\hline & SC1117 X 1206 & 23.07 & 0.51 & (-)High X (-)High & SC1117 X 1133 & 26.27 & 1.01 & (-)High X (+)High \\
\hline & SC1112 X 1205 & 24.20 & 0.45 & (+)High X (-)High & SC1112 X 1133 & 26.77 & 0.71 & $(+)$ High X (+)High \\
\hline \multirow[t]{3}{*}{ MV } & SC1112 X 1206 & 3.60 & -0.36 & (+)High X (+)High & SC1117 X 1205 & 3.37 & -0.28 & $(+)$ High X (+)Low \\
\hline & SC1117 X 1115 & 3.50 & -0.32 & (+)High X (-)High & SC1132 X 1115 & 2.80 & -0.17 & (-)High X (-)High \\
\hline & SC1117 X 1205 & 3.47 & -0.21 & (+)High X (-)High & SC1112 X 1115 & 3.10 & -0.12 & (+)High X (-)High \\
\hline
\end{tabular}

The study from Alkuddsi et al., (2013) also substantiates the current findings on relative contribution of component factors in line $\mathrm{x}$ tester mating design.

\section{General combining ability (gca) effects of parents}

General combining ability (gca) is defined as average performance of a line in a series of crosses. Best possible line and tester among the set of parents for maturity, phenological, yield, and yield component and fibre quality traits were identified under the contrasting plant densities (Table 6). From this compilation it is quite evident none of the line (female) or tester (male) harbored significant favorable general combining ability effects for all the traits under the investigation. These findings are in line with the studies of Patil et al., (2017), Talpur et al., (2016) and Karademir et al., (2009). In order to select common line (female) and tester (male) under contrasting plant densities offering significant effect for majority of traits, gca values were converted into scores as suggested by Alkuddsi et al., (2013). And also categorized the scores into three classes (Table 7) viz., significantly favorable direction, and nonfavorable direction and non-significant for each of the parents to access the overall impact. Through this analysis SC1134 and SC1205 emerged as relatively best line (female) and tester (males) respectively under both the planting densities indicating the resilience and broad based utility for the genetic improvement of corresponding traits.

\section{Specific combining ability (sca) effects of test crosses}

Specific combining ability (sca) is defined as the deviation in the performance of hybrids from the expected productivity based upon 
the average performance of lines involved in the hybrid combination. To facilitate the selection of test crosses with high sca effects in favorable direction, top three combinations in terms of magnitude were compiled for all the traits under the study (Table 8 and 9). Along with this gca status of corresponding parental line in terms of high or low and direction of utility + or - was captured to select the complimentary parents. Similar to gca effects none of the combination combined the significantly high gca effects in favorable direction for all the traits. This corroborates with the findings of Ashokkumar et al., (2010) and Preetha et al., (2008). Hence sca effect and mean per se performance for economically important trait seed cotton yield per hectare (SCYPH) was considered as criterion to identify potential cross combinations for heterosis breeding.

Under conventional density planting (CDP) test cross combination SC1104 X 1205 recorded significantly high magnitude of sca effect (255.2) with mean seed cotton yield of $3108 \mathrm{~kg} / \mathrm{ha}$. Wherein, (+) high X (-) low gca status of corresponding parents as concerned parents found be involved in the manifestation high sca effect and per se performance. Similar kind of phenomenon was observed by Alkuddsi et al., (2013). Whereas SC1134 X SC1133 test cross combination found to be having high sca effect (382.2) with per se seed cotton yield of $4343 \mathrm{~kg} / \mathrm{ha}$ under high density planting and quite contrarily, (+) high X (-) high gca status of parents led to this superior sca effect. This quite contrasting gca status of parents resulting in cross combinations with high sca effects also reported by Srinivas et al., (2014). From this study it is quite evident that, irrespective of planting densities at least one parent with high gca effect in favorable direction should be considered to improve the magnitude of sca effect and per se mean value for seed cotton yield.
The current study unveiled the possible gene action (additive and non-additive) underlying the traits associated with maturity, plant architecture, yield and fibre quality. This information could be useful to device the breeding schema for the genetic enhancement of these traits. Through the combining ability analysis, line (female) SC1134 and tester (male) SC1205 found to be good general combiner for the majority of the traits with broader utility, which could be further explored as a potential parent to breed versatile hybrids for contrasting plant densities. And also identified test cross combinations SC1104 X 1205 and SC1134 X SC1133 with high magnitude of sca effects under conventional density planting (CDP) and high density planting (HDP) respectively. These combinations can be considered as potential F1 hybrid crosses to improve yield potential through heterosis breeding.

\section{Acknowledgements}

Acknowledgment to M/S Sri Satya Agri Biotech limited, Guntur, Andra Pradesh for sharing the parental lines.

\section{References}

Alkuddsi, Y. A., Gururaja Rao, M.R., Patil, S.S., Gowda, T.H. and Joshi, M (2013). Combining ability analysis for seed cotton yield (kapas yield) and its components in intra hirsutum hybrids and forming heterotic boxes for exploitation in cotton. Genomics and Applied Biology. 4(5), 35-49.

Anonymous (2017). Status paper on cotton, Directorate of cotton development, Government of India.17-31.

Ashokkumar, K., Ravikesavan, R. and Prince, K.S.J. (2010) Combining Ability Estimates for Yield and Fibre Quality Traits in Line $\mathrm{X}$ Tester Crosses of Upland Cotton, (Gossypium hirsutum). 
Int. J. of biology. 2(1):179-189.

Dhamayanthi, K.P.M. and Rathinavel, K (2017). Heterosis and combining ability studies in extra-long staple interspecific ( $G$. hirsutum x G. barbadense) hybrids of cotton. Electronic J. Plant Breeding, 8(2): 494-500.

Gezahegn, B., van Rensburg, J. B. J. and van Deventer, C. S (2010). Heterosis and combining ability of drought-tolerant maize lines for grain yield in contrasting moisture and plant density environments. Ethiop. J. Agric. Sci. 20:151-1

Karademir, C., Karademir, E., Ekinci, R. and Gencer, O. (2009). Combining ability estimates and heterosis for yield and fiber quality of cotton in line $\mathrm{x}$ tester design. Not. Bot. Hort. Agrobot. Cluj. 37(2), 228-233

Kempthrone, O (1957). An Introduction of Genetics Statistics. The Iowa University Press.

Patil, N. P., Salve, A. N. and Adsare, A. D. (2017). Combining ability studies over environments for sucking pest and yield in upland cotton. J. Global Biosciences. 6(4): 4918-4934.

Preetha, S. and Raveendran. T.S. (2008). Combining ability and Heterosis for yield and fibre quality traits in Line $X$ Tester crosses of upland cotton
(Gossypium hirsutum L.). Int. J. Plant. Breed. Genet, 1: 1-11.

Singh, R.K. and Chaudhary, B. D (1979).Biometrical Methods in Quantitative Genetic Analysis. Kalyani Publishers, Ludhiana: 191-200.

Sprague, G.F. and Tatum, L.A. (1942) General combining ability in single crosses of corn. Agronomy, 34, 923932.

Srinivas, B., Bhadru, D., Brahmeswararao, M.V. and Gopinath, M (2014).Combining ability studies for yield and fibre quality traits in upland cotton (Gossypium hirsutum L.). SABRAO J Breed. Genetics 46 (2) 313318.

Talpur, M. Y. M., Memon, S., Mari, S.N., Laghari, S., Soomro, Z. A., Arain, S., Dev, W., Abro, A. A. and Abro, S (2016). J Basic Appl. Sci., 12, 378-382.

Vasal, S.K., Srinivasan, G., Han, G.C., Gonzales, C. F. (1992) Heterotic patterns of eighty-eight white subtropical CIMMYT maize lines. Maydica, 37, 319-327.

Yuksel, B., Hatice C. and Adem, B (2010). Combining ability and Heterosis for fibre quality traits. Plant Breed. Seed Sci., 62: 1-16 (2010).

\section{How to cite this article:}

Gowtham Prakash, S.L. Korekar and Sandeep Mankare. 2018. Combining Ability Analysis in Bt Cotton (Gossypium hirsutum L.) to Harness High Yield under Contrasting Planting Densities through Heterosis Breeding. Int.J.Curr.Microbiol.App.Sci. 7(02): 1765-1774. doi: https://doi.org/10.20546/ijcmas.2018.702.214 